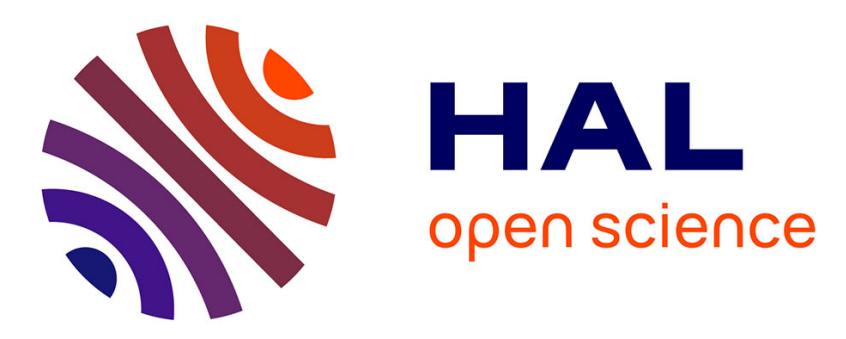

\title{
Nonlocality and Magnetic Flux Sensitivity of Superconducting Mesoscopic Aluminium Loops
}

C. Strunk, V. Bruyndoncx, C. van Haesendonck, V. Moshchalkov, Y. Bruynseraede, R. Jonckheere

\section{- To cite this version:}

C. Strunk, V. Bruyndoncx, C. van Haesendonck, V. Moshchalkov, Y. Bruynseraede, et al.. Nonlocality and Magnetic Flux Sensitivity of Superconducting Mesoscopic Aluminium Loops. Journal de Physique IV Proceedings, 1996, 06 (C3), pp.C3-253-C3-257. 10.1051/jp4:1996338 • jpa-00254256

\section{HAL Id: jpa-00254256 https://hal.science/jpa-00254256}

Submitted on 1 Jan 1996

HAL is a multi-disciplinary open access archive for the deposit and dissemination of scientific research documents, whether they are published or not. The documents may come from teaching and research institutions in France or abroad, or from public or private research centers.
L'archive ouverte pluridisciplinaire HAL, est destinée au dépôt et à la diffusion de documents scientifiques de niveau recherche, publiés ou non, émanant des établissements d'enseignement et de recherche français ou étrangers, des laboratoires publics ou privés. 


\title{
Nonlocality and Magnetic Flux Sensitivity of Superconducting Mesoscopic Aluminium Loops
}

\author{
C. Strunk, V. Bruyndoncx, C. Van Haesendonck, V.V. Moshchalkov, Y. Bruynseraede and \\ R. Jonckheere* \\ Laboratorium voor Vaste-Stoffysica en Magnetisme, Katholieke Universiteit Leuven, Celestijnenlaan \\ 200 D, 3001 Leuven, Belgium \\ * Interuniversity Microelectronics Center, Kapeldreef 75, 3001 Leuven, Belgium
}

\begin{abstract}
We have measured the magnetoresistance and the magnetic phase boundaries $T_{\mathrm{c}}(B)$ of mesoscopic superconducting structures containing an Al loop. The structures were designed to study the nonlocal interaction between the 1oop and the electrical leads connected to it. Several voltage probes have been attached to the current leads to monitor the superconducting transitions of both the loop and the segments of the leads. Strong coupling effects have been found between the loop and the leads. At low magnetic fields, $T_{c}(B)$ of the lead segments reveals a pronounced oscillatory component, while the oscillation amplinude of $T_{c}(B)$ of the loop is significantly reduced when compared to the usual Little-Parks effect. The coupling strength seems to be controlled by the temperature dependent superconducting coherence length.
\end{abstract}

\section{INTRODUCTION}

Mesoscopic superconducting structures are of strong interest as potential new components for the low temperature electronics. The quantum interference effects in small superconducting loops make them in principle suitable as flux sensitive devices.

It is well known that the magnetoconductance for mesoscopic samples obtained from a four terminal measurement is also influenced by regions of the conducting structure outside the voltage probes $[1,2]$. Due to the nonlocal nature of the electron wave function, all interference processes occuring within the phase coherence length $L_{\varphi}$ contribute to the measured conductance. The nonlocality becomes particularly obvious when a loop is connected to a line, since in that case the Aharanov-Bohm effect in the loop is reflected as an oscillatory contribution to the magnetoconductance of the line. While the nonlocal contribution to the conductance of normal metal structures is rather small (about $e^{2} / h \approx 4 \cdot 10^{-5} \Omega^{-1}$ ), very pronounced nonlocal effects should be observable just below the superconducting transition temperature $T_{c}$, where the conductance is dominated by the presence of the superconducting condensate. Here, we will concentrate on the fundamental properties of mesoscopic aluminium loops as well as their applicability as magnetic flux sensors.

We have studied square loop structures connected to electrical leads for transport measurements. A number of voltage probes attached to the leads allow the measurement of the superconducting transitions of the loop and of certain segments of the leads located at various distances from the loop. The Little-Parks effect [3] in the loop induces oscillations with flux period $\Phi_{0}=h / 2 e$ in the magnetoresistance and in the normal/superconducting phase boundary $T_{c}(B)$ of the lead segments. On the other hand, the amplitude of the Little-Parks oscillations in the loop itself is strongly reduced by the attached leads. When the superconducting coherence length $\xi(T)$ diverges near $T_{c}$, the loop and the leads are expected to be strongly coupled via the proximity effect while they should behave independently at lower temperatures where $\xi(T)$ decreases. 


\section{EXPERIMENTAL}

Samples were prepared by thermal evaporation of $99.999 \%$ pure Al onto oxidized Si wafers. The patterns were defined using standard electron beam lithography with a bilayer PMMA resist and lift-off techniques. Scanning electron and atomic force microscopy confirmed the presence of a smooth $\mathrm{Al}$ surface with no major cracks or holes down to the nanometer scale. The superconducting coherence length $\xi(T)=\xi_{G L}(1-$ $\left.T / T_{e 0}\right)^{-1 / 2}$ can be estimated using $\xi_{G L}=0.85\left(\xi_{0} l_{e l}\right)^{1 / 2}$, where $\xi_{0}=1600 \mathrm{~nm}$ is the BCS coherence length of $\mathrm{Al}, T_{\mathrm{co}}$ being the transition temperature in zero magnetic field and $l_{e l}$ the elastic mean free path. We will discuss a series of samples prepared under identical conditions during a single evaporation run. The relevant material parameters for this series of samples are the film thickness $t=43 \mathrm{~nm}$, the sheet resistance $R_{\square}=0.6 \Omega, l_{e l}=18 \mathrm{~nm}$ and $\xi_{G L}=125 \mathrm{~nm}$.

The inset in Figure 1 shows the schematics of a typical Al structure. It consists of $0.13 \mu \mathrm{m}$ wide strands, interrupted by a square loop of $1 \mu \mathrm{m}$ outer diameter. With our design we can measure the voltage across a $1 \mu \mathrm{m}$ segment of the leads on both sides of the loop as well as the voltage across the loop itself. The distance $L$ between the inner voltage probes and the loop is varied between 0.2 and $2.0 \mu \mathrm{m}$. The linewidth of the current and voltage leads is kept constant at $0.13 \mu \mathrm{m}$ up to a distance of $7 \mu \mathrm{m}$ from the sample to minimize the influence of the wider parts of the contact leads on the measurement. The magnetic field is always applied perpendicular to the sample.

The transport measurements have been performed using a PAR 124A lock-in amplifier for ac measurements at $27 \mathrm{~Hz}$ and measuring currents of 50 and $100 \mathrm{nA}$ rms. All electrical leads are shielded by pi filters with a cut-off frequency of $1 \mathrm{MHz}$. The normal/superconducting phase boundaries have been measured by tracing the midpoint temperature of the resistive transition with the aid of a feedback technique while ramping slowly the magnetic field. The temperature stability of the feedback circuit was about $0.1 \mathrm{mK}$.

\section{RESULTS}

In Figure 1 the measured normal/superconducting phase boundaries $T_{c}(B)$ of a loop and a lead segment at a distance $L=0.2 \mu \mathrm{m}$ to the loop are shown. The loop shows the expected Little-Parks oscillations with a field period $\Delta B=2.7 \mathrm{mT}$ corresponding to one flux quantum in the loop as estimated from the loop size. We observe an unexpected and pronounced increase of the oscillation amplitude $\Delta T_{c}(B)$ with increasing field. Previous measurements on $\mathrm{Al}$ microcylinders [4] have revealed a field independent oscillation amplitude, which is also in agreement with the theoretical model calculations [5]. On the other hand, the lead segment clearly shows oscillations at low fields which, are rapidly vanishing when the field is increased.

The solid and dashed lines in Fig. 1 correspond to the theoretical phase boundaries of isolated loops and leads, respectively. For a superconducting microcylinder in an axial magnetic field one obtaines from the linearized Ginzburg-Landau theory [4]:

$$
T_{c}(\Phi)=T_{c 0}\left\{1-\left(\frac{\xi_{G L}}{R_{m}}\right)^{2}\left[\left(\frac{\Phi}{\Phi_{0}}\right)^{2}\left(1+z^{2}\right)-2 n \frac{\Phi}{\Phi_{0}}+\frac{n^{2}}{2 z} \ln \left(\frac{1+z}{1-z}\right)\right]\right\} .
$$

Here, $\Phi=\pi R_{m}^{2} B$ is the magnetic flux enclosed by the loop, $R_{m}$ being the effective radius of the loop, $d$ the linewidth and $z=d / 2 R_{m}$ its aspect ratio. The integer $n$ has to be chosen to maximize $T_{c}(\Phi)$ for a given value of $\Phi$. The envelope of $T_{c}(\Phi)$ for a cylinder is identical to the phase boundary $T_{c}(B)$ of a thin film in a parallel magnetic field [5] :

$$
T_{c}(B)=T_{c 0}\left[1-\frac{\pi^{2}}{3}\left(\frac{d \xi_{G L} B}{\Phi_{0}}\right)^{2}\right]
$$

For mesoscopic samples Eqs. (1) and (2) remain valid for perpendicular fields, provided $\xi(T) \gg d[6]$. This condition is always fulfilled in the temperature range under consideration. When $d$ is known, $\xi_{G L}$ can 
be determined by fitting Eq. (2) to the measured envelope of the phase boundaries. Using the linewidth determined via electron microscopy, we find values for the Ginzburg-Landau coherence length which vary within a series of samples by not more than $4 \%$ and are in good agreement with the $\xi_{G L}$ values obtained from the mean free path $l_{e l}$. Since $\xi_{G L}$ is determined by the monotonic background, the theoretical oscillation amplitude for an isolated loop is not a free parameter and can be directly compared to the experimental data for the loop with attached leads. When the field is increased, the oscillation amplitude approaches the theoretical value.

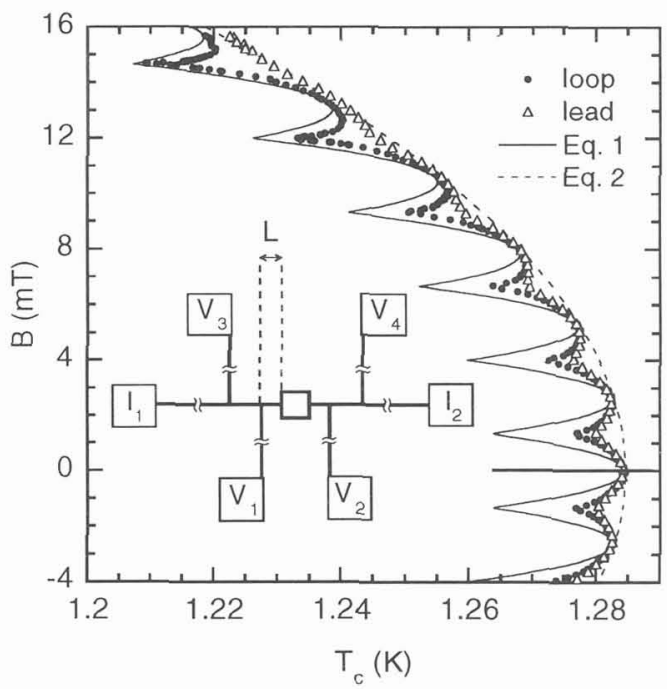

Fig. 1: Magnetic phase boundaries of the loop (voltage contacts $V_{1} / V_{2}$ ) and one of the lead segments (voltage contacts $\left.V_{1} / V_{3}\right)$ for $L=0.2 \mu \mathrm{m}$ distance between the loop and the line segment. The inset shows a schematic of the sample layout.

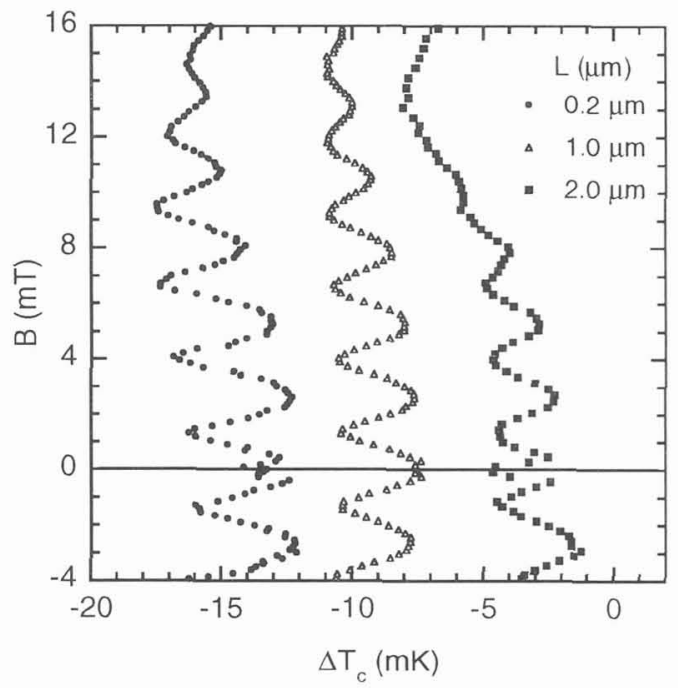

Fig. 2: Nonlocal contribution to $T_{c}(B)$ of the line segments. The parabolic background contribution due to the finite linewidth (Eq. 2) has been subtracted for clarity.

To illustrate the nonlocal effect of the loop on the leads, the phase boundaries of a series of samples with increasing distance $L$ between the measured lead segment and the loop are shown in Fig. 2. The transition temperature of the leads clearly oscillates as a function of $B$. In order to better resolve these smaller oscillations in the lead segments, we have subtracted from the measured $T_{c}(B)$ curves the monotonic parabolic background described by Eq. (2). The oscillation amplitude decreases with increasing field until it disappears in the experimental background noise. The oscillations gradually disappear at smaller fields when the distance between the measured line segment and the loop increases.

Experimental evidence for the nonlocal Little-Parks effect had already been reported in [7]. However, the latter study has been complicated by the presence of strong resistance anomalies at low fields $\dot{B} \leq$ $1 \mathrm{mT}$. At these low fields the resistance is strongly enhanced and can even exceed the normal state resistance. These anomalies, which are related to nonmonotonic transitions in $R(T)$, have been observed in mesoscopic aluminium samples of different geometry during the past few years [8,9]. Very recent experiments [10] indicate that the anomalies can be suppressed by proper radio frequency filtering of the electrical leads. A possible explanation for the anomalies in terms of charge imbalance around phase-slip centers in the small samples has been proposed $[10,11]$. In Fig. 2 small low-field anomalies are still present, which are probably induced by residual rf radiation.

It can be seen from Eq. (2) that at the phase boundary the magnetic field and $\xi(T)$ are related by: $1 / \xi(T)=(\pi d) /\left(\sqrt{3} \Phi_{0}\right) \cdot B$. Hence, the change of the oscillation amplitude with increasing magnetic field directly reflects the dependence of the coupling strength on the coherence length. In Fig. 3 the os- 
Fig. 3: Oscillation amplitude $\Delta T_{c}(B)$ versus inverse coherence length for a loop (top panel) and the lead segments from Fig. 2 (bottom panel). Note the different scales for the $\Delta T_{c}$ axes.

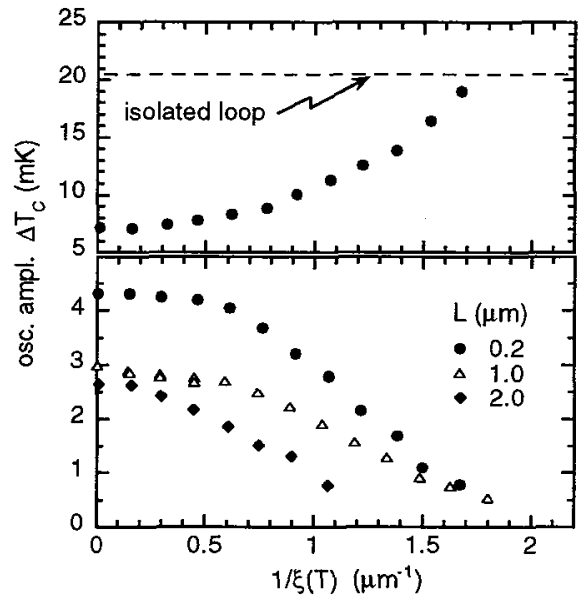

cillation amplitudes $\Delta T_{c}$ for the loop (top panel) and different lead segments (bottom panel) are plotted as a function of $1 / \xi(T) . \Delta T_{c}$ of the loop increases with increasing $1 / \xi(T)$ as opposed to $\Delta T_{c}$ of the lead segments. The dashed line in the top panel of Fig. 3 corresponds to the the limiting case of the oscillation amplitude of an isolated loop with the same $\xi_{G L}$.

\section{DISCUSSION}

The results presented above demonstrate that the loop cannot be considered separately from the leads connected to it. While the transition temperature of an isolated loop oscillates with a constant amplitude as a function of the magnetic field, the phase boundaries $T_{c}(B)$ of the leads should be monotonic in the absence of the nonlocality. Connecting the loop to the leads has an effect on both of them - the oscillations in the loop are strongly damped and $T_{c}(B)$ of the leads reveals an oscillatory component. As inferred from the observed temperature dependence of the effect, this phenomenon can be linked to the stiffness of the superconducting order parameter. In contrast to an isolated loop or a microcylinder, the loops in our structures remain superconducting until the pair breaking by the circular current, which is required to maintain the fluxoid quantization in the loop, is sufficient to drive a certain fraction of the leads into the normal state as well. As illustrated in Fig. 3, the length scale determining the coupling strength is indeed the coherence length $\xi(T)$. The problem of a loop with attached leads has been treated theoretically in the limit of vanishing linewidth - Fink et al. $[12,13]$ have found that the leads indeed reduce the oscillations amplitude of $T_{c}(B)$ of the loop when compared to an isolated loop. However, the theory overestimates the coupling strength by assuming a single transition temperature for the whole structure. This assumption cannot hold, when the strands are much longer than $\xi(T)$. As confirmed in Fig. 1, the background depression of $T_{c}$ due to the finite linewidth is still negligible for the first oscillation period. Even in this case we find that the difference between the transition temperatures of the loop and the leads is nonzero though considerably smaller than the Little-Parks effect of an isolated loop.

A strictly one-dimensional theory can also not account for the observed crossover from coupled to decoupled behavior, since the decoupling is induced by the background depression of $T_{c}(B)$ due to the finite linewidth. Once $T_{c}$ has been sufficiently decreased by the magnetic field $\xi(T)$ becomes smaller than the loop size, the coupling weakens and the experimental phase boundaries gradually approach the behavior of the isolated systems.

The flux sensitivity of our small superconducting loops is also reflected by the critical current $I_{c}(B)$ of the loops. Close to the transition temperature a SQUID-like modulation of $I_{c}(B)$ is found, although 
the loops do not contain any artificial weak links [14]. In near future this SQUID-like behavior may be exploited for the design of small and simple magnetic field sensors, which are suitable for magnetization measurements on single mesoscopic samples.

\section{CONCLUSIONS}

We have shown that a mesoscopic superconducting loop and the leads connected to it form a strongly coupled system. The mutual nonlocal influence is due to the divergence of the coherence length near the transition temperature and is suppressed at lower temperatures. Although simple one-dimensional models cannot account for all of our observations, a qualitative interpretation in terms of the proximity effect is possible.

\section{Acknowledgements}

This work has been supported the Belgian Inter-University Attraction Poles (IUAP) and the Flemish Concerted Action (GOA) programs, as well as by the Belgian National Fund for Scientific Research (NFWO).

\section{References}

[1] C. P. Umbach et al., Appl. Phys. Lett. 50, 1289 (1987).

[2] A. Benoit et al., Phys. Rev. Lett. 58, 2343 (1987).

[3] W. A. Little and R. D. Parks, Phys. Rev. Lett. 9, 9 (1962).

[4] R. P. Groff and R. D. Parks, Phys. Rev. 176, 567 (1968).

[5] M. Tinkham, Phys. Rev. 129, 2413 (1963).

[6] V. V. Moshchalkov et al., Nature 373, 319 (1995).

[7] N. E. Israeloff, F. Yu, A. M. Goldman, and R. Boiko, Phys. Rev. Lett. 71, 2254 (1993).

[8] P. Santhanam et al., Phys. Rev. Lett. 66, 2254 (1991).

[9] H. Vloeberghs et al., Phys. Rev. Lett. 68, 1268 (1992).

[10] C. Strunk et al, to be published in Phys. Rev. B (May 1996).

[11] M. Park, M. S. Isaacson, and J. M. Parpia, Phys. Rev. Lett. 75, 3740 (1995).

[12] H. J. Fink, A. López, and R. Maynard, Phys. Rev. B 26, 5237 (1982).

[13] S. B. Haley and H. J. Fink, Phys. Lett. 102A, 431 (1984).

[14] V. V. Moshchalkov et al., Nature 361, 617 (1993). 